\title{
Particle imaging through planar shock waves and associated velocimetry errors
}

\author{
G. E. Elsinga ${ }^{1}$ G. C. Orlicz ${ }^{2}$
}

Received: 10 March 2015 / Revised: 22 April 2015 / Accepted: 26 May 2015 / Published online: 6 June 2015

(C) The Author(s) 2015. This article is published with open access at Springerlink.com

\begin{abstract}
When imaging particles through a shock wave, the resulting particle image appears blurred and at the wrong location, which is referred to as a position error. Particle image doublets are observed if only part of the light scattered by a particle is deflected or reflected by the shock. These optical distortions are due to the jump in the refractive index that occurs over the shock. Within the context of popular particle-based velocimetry techniques, such as particle image velocimetry and particle tracking velocimetry, the position error propagates into an error in the measured velocity. These particle image distortions and associated errors are assessed and quantified in this paper for the case of planar shocks by means of a light ray tracing approach and by experiments. The errors are shown to be most sensitive to the angle between the viewing direction and the plane of the shock. Increasing this angle to modest values $\left(\sim 5^{\circ}\right)$ is a particularly effective way to decrease the relative velocity error. Looking at the shock from the high-density side is recommended when the accurate determination of the particle response to the shock wave is desired.
\end{abstract}

\section{Introduction}

Compressible flows are well known to distort light rays propagating through it. In fact for many years, these

G. E. Elsinga

g.e.elsinga@tudelft.nl

1 Laboratory for Aero and Hydrodynamics, Department of Mechanical, Maritime and Materials Engineering, Delft University of Technology, Leeghwaterstraat 21, 2628 CA Delft, The Netherlands

2 Physics Division, MS H803, Los Alamos National Laboratory, Los Alamos, NM 87545, USA principles have been used to visualize and study such flows (i.e., shadowgraphy, schlieren methods and interferometry, Merzkirch 1974; Settles 2001). Particle image velocimetry (PIV) and particle tracking velocimetry (PTV) being optical techniques are affected by similar aero-optical distortions resulting in particle image blur and introducing error in the measured particle velocity (Raffel and Kost 1998; Hou et al. 2002; Schrijer et al. 2005). These errors were quantified and described in detail by Elsinga et al. (2005) for flows with stationary and smoothly varying density distributions, hence continuous refractive index fields. An example of optical distortion in a highly instationary compressible flow is given in Murphy and Adrian (2010).

Here, we extend our earlier work to the special case of planar shock waves, over which the refractive index is discontinuous. Indeed, the jump in the refractive index across the shock can cause significant PIV measurement error and leads to interesting particle imaging phenomena. Based on the analysis, we provide recommendations to deal with the particle displacement error. The work is motivated by the need to accurately measure the particle velocity fields across a shock when estimating the particle response time (Scarano and Van Oudheusden 2003; Schrijer and Scarano 2007) and by the recent interest in inertial particle and droplet dynamics in supersonic flows with shock waves (Buchmann et al. 2014; Martinez et al. 2015). Compared to other optical interfaces in fluids, such as occurring in two-phase flow, shock waves are characterized by a small jump in refractive index (order $10^{-4}$ ) and fluid flowing through it at a significant relative velocity. This means light is deflected over small angles so that the recordings still reveal recognizable particle images, albeit clearly distorted. At the same time, the velocity errors can be considerable.

The theory of particle imaging across planar shocks is examined first taking a light ray tracing approach, which 
gives insight into the blurring phenomenon and provides estimates for the particle image position error and velocity error (Sect. 2). The errors are shown to depend strongly on the viewing direction relative to the plane of the shock. The predictions are then compared with results from PTV and PIV measurements (Sects. 3, 4), followed by an assessment of the particle response estimated from the velocity measurements (Sect. 5). The findings are summarized in Sect. 6.

\section{Theory}

The aero-optical distortion is examined first by means of numerical simulations, in which we consider a stationary shock wave with the particles moving relative to the shock. The general layout of the simulated experiment is presented in Fig. 1b (right column). The planar shock is at an angle $\theta$ with the viewing direction and terminates at the wind tunnel window. The density $\rho$ increases over the shock corresponding to a jump in refractive index from $n_{1}$ to $n_{2}>n_{1}$ according to the Gladstone-Dale relation; i.e., $n=1+K \rho$, where $K=2.3 \times 10^{-4} \mathrm{~m}^{3} / \mathrm{kg}$ for air. The distance between the PIV light sheet and the tunnel window is $W$. The light that is scattered by a particle and captured by the imaging optics forms a cone, which solid angle depends on the selected objective $f / \#$ and magnification $M$ (as shown later in Eq. 6). Light rays within this cone are traced from the particle to the imaging optics. In case a light ray intersects with the shock, Snell's law applies and the deflection angle can be calculated given the intersection angle and the jump in refractive index. Total reflection occurs if the particle is on the high-density side and the angle between the ray and the shock plane is below the critical angle, which is given by $\theta_{\text {crit }}=\sqrt{2\left(n_{2}-n_{1}\right)}$ for small $\theta$. The ray trace data determine the geometrical shape of the particle image. Then, the final particle image is obtained after a convolution with the diffraction spot, which is again a function of $f / \#$ and $M$ (Raffel et al. 1998). Simulations are carried out varying the particle positions relative to the shock and changing the viewing angle $\theta$ and the $f / \#$. Here, the viewing angle $\theta$ is defined as the angle between the plane of the shock and the viewing direction, or the optical axis. The layout of the optical arrangement is consistent with the wind tunnel experiments to be presented later. Generally, the jump in refractive index over the shock wave would also deflect the light sheet. However, light propagation within the sheet is typically in the direction normal to the shock (or close to), in which case it is easy to show from Snell's law that the deflection angle is insignificant. Therefore, we will not consider the optical distortion of the light sheet in this paper.

Based on the simulation results, the particle images can be categorized into four types depending primarily on the viewing angle $\theta$ and the position of the particle relative to the shock (Fig. 1). The first type is the undistorted particle image, which is formed when none of the light rays intersect the shock (Fig. 1a). The second type results when all rays cross the shock (Fig. 1b). The distorted particle image is characterized by an asymmetrical stretching in the direction normal to the shock (referred to as skewed directional blurring). The blur length is linear with the distance between the particle and the shock, because the incidence angles of all light rays with the shock, and consequently the deflection angles, do not change with the position of the particle. Type III occurs when only part of the rays intersect the shock (Fig. 1c). In that respect, it can be considered an end-effect resulting from the shock being finite in the viewing direction. Interestingly, particle doublets can be observed in this case. The particle on the right in Fig. 1c is the sharply imaged undistorted component, and the blurred doublet part on the left is the result of refraction by the shock. The blurred doublet part is shifted toward the lowdensity side relative to the undistorted part of the particle image. With type IV, the particle needs to be located on the high-density side and the light rays are required to intersect the shock at very small angles, which are below the limit angle for total internal reflection. For example, the critical angle between the shock plane and the light ray is only $0.7^{\circ}$ when the density jumps from 0.6 to $0.9 \mathrm{~kg} / \mathrm{m}^{3}$, corresponding to a change in refractive index of $n_{2}-n_{1}=0.6 \times 10^{-4}$. The shock acts as a mirror, and the doublet is also sharply imaged (Fig. 1d). Although both types of doublets (III and IV) may appear similar, there are some fundamental differences. First, the velocity of the doublet due to reflection has the opposite direction to that of the actual particle! The shock mirrors not only the particle image but also its displacement. Secondly, the refraction-type doublet (III) always appears in the image on the same side of the shock as the actual particle, whereas the reflection-type doublet (IV) appears on the opposite side of the shock; i.e., the reflected part appears on the low-density side of the shock when the undistorted part of the particle image is on the high-density side. Both doublets appear only under very specific conditions, but they can be a source of uncertainty when tracking individual particles (e.g., PTV) or contribute to a loss of correlation in PIV.

From the ray traces in Fig. 1b, c, it is evident that the blurred particle (type II as well as the blurred doublet part in type III) appears in the image at a position different to its true location. This difference is referred to as a particle position error $\xi$ (Elsinga et al. 2005). Thus, particle image blur and position error are concurrent. In order to evaluate the position error $\xi$, it is convenient to define a spatial coordinate $s$ relative to the shock (Fig. 1b, right column). Within the light sheet, $s$ is the distance from the intersection of the light sheet with the shock and is positive toward the high-density side. In the direction along the 
(a) type I: undistorted particle image
Occurs when all rays do not intersect with the shock (reference case)

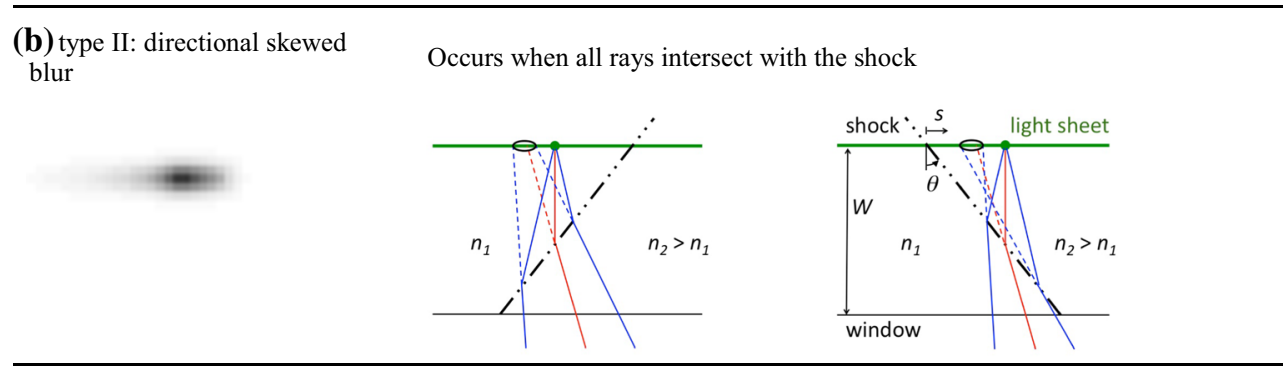

(c) type III: doublet (refraction)

Occurs when only part of the rays intersect with the shock

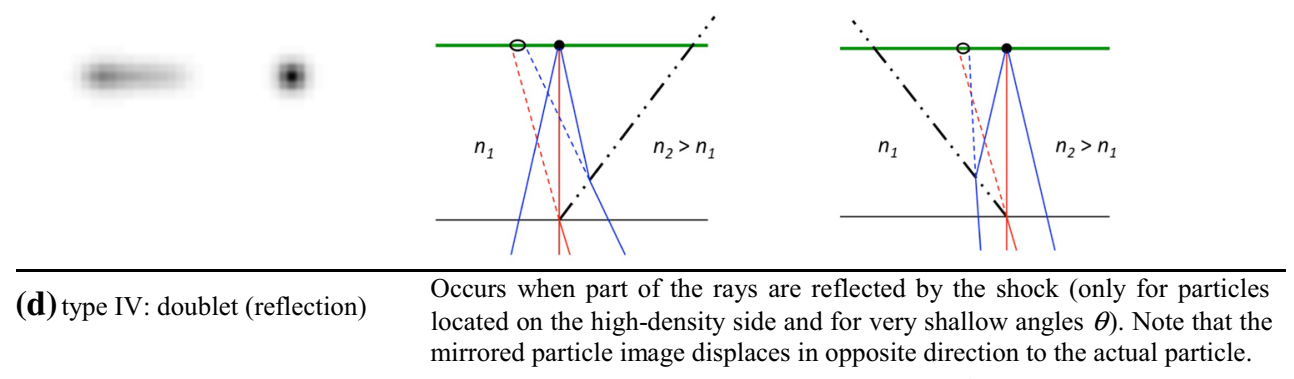

Fig. 1 Four types of particle images near shock waves (a-d). The left column presents the resulting image (inverted gray scale). The middle and right columns schematically show the distortion of light rays by the shock for viewing angles $\theta<0$ and $\theta>0$, respectively. The dash-dotted line indicates the planar shock with the high-density side to its right. The shock terminates at the tunnel window. The blue and red solid lines are light rays originating from the actual particle and propagating toward the imaging system outside the flow (not shown). The blue rays bound the light cone captured by the optics, which top angle is set by $f / \#$ and $M$ (Eq. 6). The dashed lines are backward extensions of the distorted light rays, which indicate the apparent origin of the ray within the light sheet (i.e., plane of focus) as perceived by the imaging system. They can thus be used to construct the recorded particle image at the location of the light sheet shock wave, the refractive index is uniform so that, for a given shock, the optical distortion depends only on $s$. Furthermore, the angles $\theta$, as well as the light ray deflection angles, are assumed to be small. Then, based on the ray at the light cone axis (the red line in Fig. 1b), the perceived position error $\xi(s)$ for a particle located at $s$ is estimated as:

$$
\begin{aligned}
& \text { if } \theta<0: \\
& \xi(s)= \begin{cases}{\left[-1+\sqrt{\left.1+\frac{2\left(n_{2}-n_{1}\right)}{\theta^{2}}\right] s} \text { for } W \tan (\theta)<s<0\right.} \\
0 & \text { elsewhere }\end{cases} \\
& \text { if } \theta>\sqrt{2\left(n_{2}-n_{1}\right)}: \\
& \xi(s)= \begin{cases}{\left[-1+\sqrt{1-\frac{2\left(n_{2}-n_{1}\right)}{\theta^{2}}}\right] s} & \text { for } 0<s<W \tan (\theta) \\
0 & \text { elsewhere }\end{cases}
\end{aligned}
$$

where $n_{1}$ and $n_{2}>n_{1}$ are the refractive indices upstream and downstream of the shock, respectively. The cases $\theta<0$ (Eq. 1) and $\theta>0$ (Eq. 2) correspond to the schematics in the middle and right column of Fig. 1b, c, respectively. Note that $\xi$ is linear in $s$ and is always negative, meaning that the blurred particle image is shifted in the direction of decreasing $s$ (i.e., toward the low-density side) relative to the true particle position. However, the blurred particle image due to refraction must appear on the same side of the shock as the actual particle, as mentioned before. The maximum position error magnitude is reached when $s=W \tan (\theta)$, which is associated with the maximum distance between the shock and the particle measured along the light ray/optical axis and corresponds to the location where the shock terminates at the tunnel window. Refraction-type doublets (type III, 
Fig. 2 Particle position error $\xi$ (top) and relative velocity error $\varepsilon_{s}$ (bottom row) for negative (left) and positive angles $\theta$ (right column). In the $\varepsilon_{s}$ graphs, the blue lines represent the spatial derivative of the position error (Eq. 4), while the red lines illustrate the effects of finite cross-correlation window size and finite-particle displacement between consecutive images, which are approximated by a moving average filter
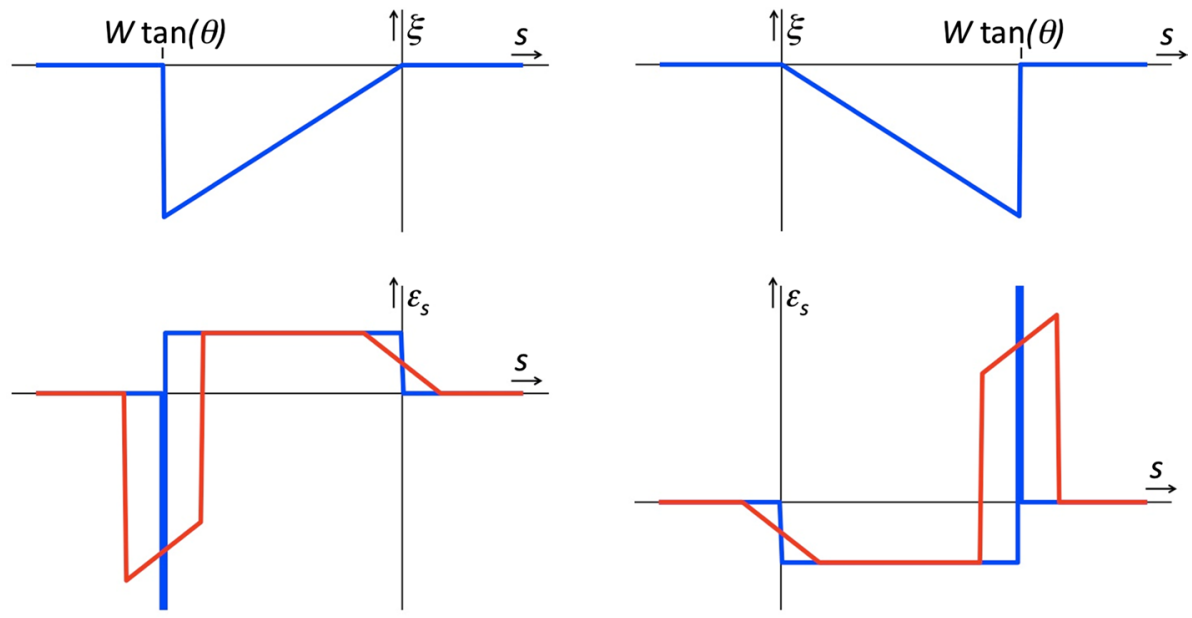

Fig. 1c) occur around the same $s$ position. In this case, the sharply focused part corresponds to the undistorted particle image, while the blurred doublet part forms from the refracted light, which is shifted with respect to the undistorted image over a distance $\xi(s \approx W \tan (\theta))$. The distance between both parts of the refraction doublet may thus be indicative of the maximum position error.

Furthermore, the position error introduces an error in the observed particle velocity in the direction of $s$. This component of velocity is denoted by $V_{s}$, and its relative error by $\varepsilon_{s}$. From the general equation for the velocity error due to aero-optical distortion (Eq. 6 in Elsinga et al. 2005), the following expression is obtained for the component $\varepsilon_{s}$ :

$\varepsilon_{s}=\frac{\Delta V_{s}}{V_{s}}=\frac{\partial \xi}{\partial s}-\frac{1}{V_{s}} \frac{\partial V_{s}}{\partial s} \xi$

On the right-hand side of the above equation appear two contributions. The first is the spatial gradient of the position error, which can be interpreted as a local stretching of the image. The second term is associated with a shift $\xi$ of the velocity profile. In the case of a shock, the particle velocity and the spatial gradient of the position error approximately align, which, as shown in Elsinga et al. (2005), causes the first term to dominate. Then, upon substitution of Eqs. 1 and 2, Eq. 3 simplifies to:

$\varepsilon_{s} \approx \frac{\partial \xi}{\partial s}=-1+\sqrt{1-\operatorname{sign}(\theta) \frac{2\left(n_{2}-n_{1}\right)}{\theta^{2}}}$

which is valid over the range in $s$ corresponding to nonzero position error, $\xi$, as specified in Eqs. 1 and 2. An important observation is that $\varepsilon_{s}$ turns out to be independent of the physical size of the flow, i.e., the length $W$. It is a function only of the viewing angle, $\theta$, and the jump in refractive index $n_{2}-n_{1}$. Furthermore, note that due to the discontinuity in $\xi$ at $s=W \tan (\theta)$ there exists a singularity in $\varepsilon_{s}$ at that position. This is illustrated graphically in Fig. 2. Whether such a discontinuity exists in reality is questionable. We have assumed the shock to terminate at the tunnel window without change. In a shock tube, this is a reasonable assumption, because the initial boundary layer growth after shock passage is very small (Petersen and Hanson 2003). But in a wind tunnel flow, the shock will interact with the boundary layer developing over the window (Adamson and Messiter 1980). The interaction will likely smoothen the refractive index field locally, hence the optical distortions. Moreover, the doublets that appear in the images at the location of the $\xi$ discontinuity will be of a relatively low intensity, because the light scattered by the particle is divided over two parts rather than concentrated in one single image. The fraction of the scattered light that ends up in the blurred doublet part depends on the distance relative to the $\xi$ discontinuity, which may assist in removing the singularity in a PIV correlation analysis. Furthermore, from Fig. 2, it is clear that the optical distortion errors affect the measurements on the low-density side (upstream of the shock, $s<0$ ) when looking at the shock from the highdensity side (i.e., $\theta<0$ ) and vice versa. When including effects of finite PIV spatial resolution, the relative velocity error, $\varepsilon_{s}$, exhibits a wiggle. In the direction of increasing $s$, $\varepsilon_{s}$ changes sign from negative to positive, independent of $\theta$ (Fig. 2).

The above analysis produces a significant insight in that the position error at a given $s$ and the relative velocity error drop sharply with the angle $\theta$ according to $\theta^{-2}$. Aiming at reducing the velocity errors, it can thus be beneficial to slightly increase the angle between the shock and the viewing direction. It should be noted, however, that while the errors decrease, the region in space affected by optical distortions increases according to $W \tan (\theta)$, as shown in Fig. 2. The sensitivity of the relative velocity error to the angle $\theta$ is shown in Fig. 3 for a given jump in the refractive index, which corresponds to the experimental conditions in Sect. 4. At $\theta=1^{\circ}$, the error is around $20 \%$, but it reduces to just 2.5 and $1 \%$ when 


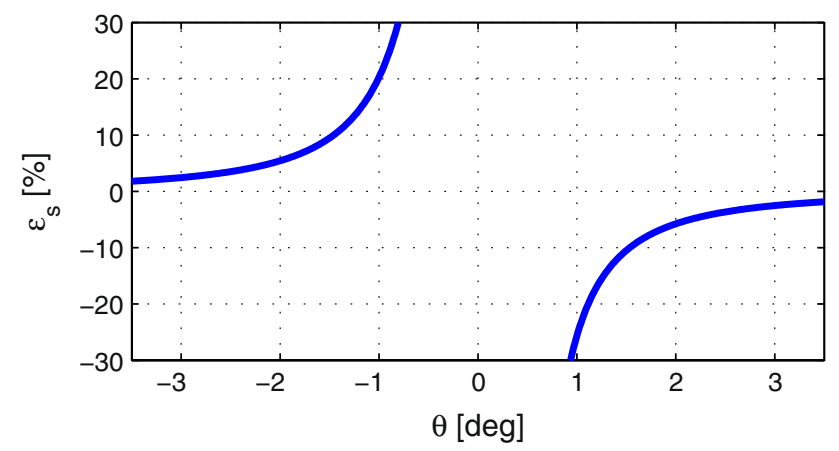

Fig. 3 Magnitude of the relative velocity error (Eq. 4) versus the angle $\theta$ between the shock and the viewing direction. In this example, the refractive index increases by $n_{2}-n_{1}=0.6 \times 10^{-4}$ over the shock

$\theta$ is increased slightly to $3^{\circ}$ and $5^{\circ}$, respectively. The fact that the error is insignificant at large angles motivates our initial choice of considering only shallow angles $\theta$ in the present analysis.

Next the geometric blur length is estimated by back propagating the two light rays bounding the light cone (blue lines in Fig. 1) to the plane of the light sheet. Again assuming small $\theta$, but with $|\theta|>\alpha$, the distance between the rays at the location of the light sheet is:

$$
\begin{aligned}
\xi_{\text {blur }}(s) & =\operatorname{sign}(\theta)\left(n_{2}-n_{1}\right)\left(\frac{1}{(\theta-\alpha)^{2}}-\frac{1}{(\theta+\alpha)^{2}}\right) s \\
& \approx \frac{4\left(n_{2}-n_{1}\right) \alpha}{\theta^{3}} s
\end{aligned}
$$

where $\alpha$ is the half top angle of the light cone, which is given by:

$\tan (\alpha)=\frac{1}{2 f_{\#}} \frac{M}{M+1}$

The above result is valid if all rays within the light cone intersect with the shock (Fig. 1b). In that case, the geometric blur length is found to increase with decreasing $\theta$ and also with decreasing $f / \#$. The present result complements the maximum blur estimate for $\theta=0$ derived by Raffel and Kost (1998), i.e., $\xi_{\text {blur }}=W \sqrt{2 \Delta n}$, which does not depend on $f / \#$. Generally, blur results in larger diameter particle images, which are expected to increase the noise level in the velocity measurement (Raffel et al. 1998).

\section{Particle imaging: experiment}

In this section, the experimental evidence for the predicted particle image distortion is considered. Eight-frame particle tracking recordings from the experimental facility described in Martinez et al. (2015) are used for this purpose because the low image seeding density allows unambiguous detection of the individual particles, while the time resolution offers the opportunity to observe the different stages of distortion as a particle passes the shock (in the shock reference frame). Both the shock tube facility and the experiment were described in detail in their paper. We briefly address the main features pertaining to the images used here.

Within the tube, a Mach 1.30 normal shock wave was produced, which propagated through the test section at $447.6 \mathrm{~m} / \mathrm{s}$. Upstream of the shock, the flow was at rest. Over the shock, the density increased from 0.93 to $1.41 \mathrm{~kg} / \mathrm{m}^{3}$ corresponding to a jump in the refractive index of $n_{2}-n_{1}=1.2 \times 10^{-4}$. The images of the 2- to $10-\mu \mathrm{m}$-diameter glass particles dispersed in the flow were recorded with $f / \#=22$ and magnification $M=2$, which resulted in a resolution of 310 pixels $/ \mathrm{mm}$ in the measurement plane. The distance between the light sheet and the tunnel window, $W$, was $38 \mathrm{~mm}$. Per run a total of 8 frames were recorded at $0.3-\mu$ s time intervals. The position of the shock wave was determined from a simultaneous shadowgraph recording to within an uncertainty of about $0.03 \mathrm{~mm}$.

To compare the experimental results with the predictions, a moving frame of reference is adopted in which the coordinate $s$, introduced in Sect. 2, is fixed to the propagating shock. Hence, the particle velocity, $V_{s}$, used when evaluating the relative velocity error (Eq. 4), is the velocity relative to shock. Another subtle difference with respect to the simulations in the previous section is the fact that the viewing angle $\theta$ changes as the shock moves across the field of view, which is related to the opening angle of the lens. During the total recording, the shock displaces by $0.94 \mathrm{~mm}$, which results in an approximate $0.4^{\circ}$ change in $\theta$.

Figure 4 presents an example of a particle upstream of the shock $(s<0)$. With time the distance to the shock decreases and the image distortions develop. The viewing direction, $\theta$, in this case is slightly negative, which we infer from the fact that the particle image is distorted just upstream of the shock. Initially, the particle image is undistorted (frame 1), but as the shock approaches some of the light rays will be deflected and a refraction-type doublet becomes apparent (frames 2-6). The distance between the undistorted part of the particle image and the blurred doublet part deceases with decreasing distance to the shock. As argued above, the distance between the two parts of the particle image is indicative of the position error due to refraction, which indeed decreases with decreasing distance to the shock (Eq. 1). When all light rays finally intersect with the shock, a skewed directional blurred particle image results (frame 7). Then after crossing the shock, the particle image again appears 


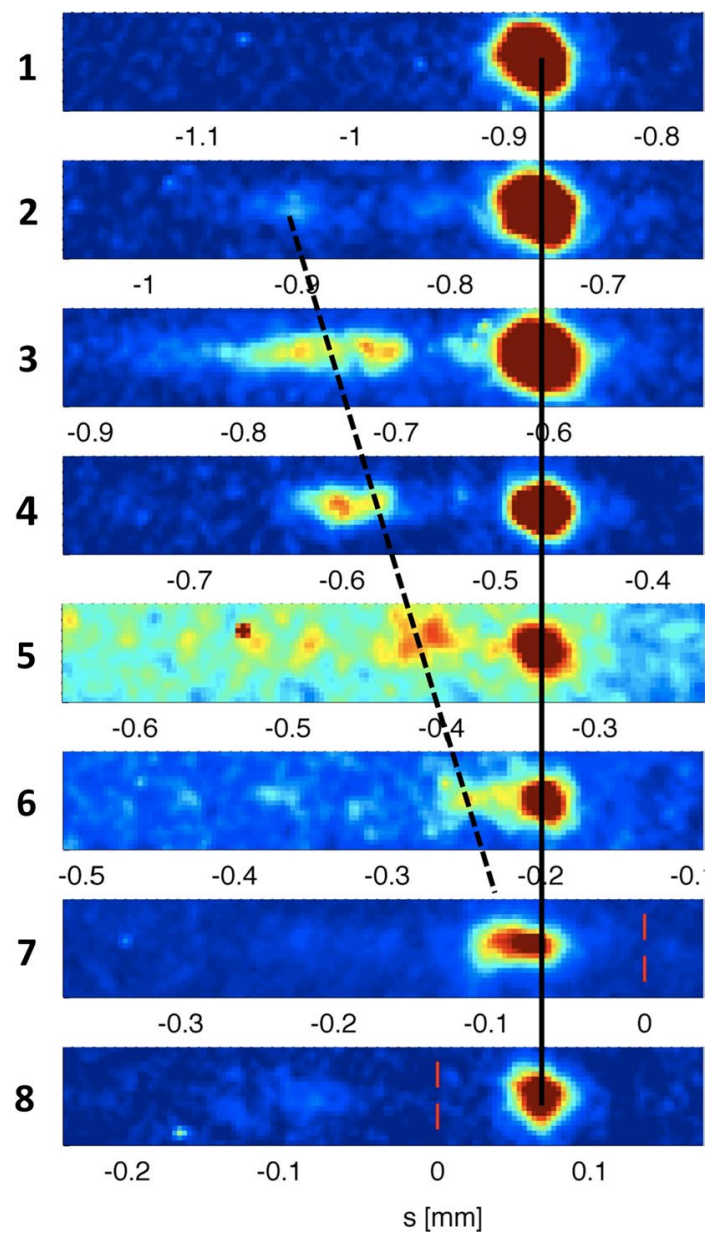

Fig. 4 Time series of a particle image showing the optical distortion at several distances upstream of the shock. Frames 2-6 show a refraction-type doublet, and frame 7 shows a skewed directional blurred image. The shock position is indicated by a red dashed line, which is visible only in frames 7 and 8 . The increased background intensity in frames 5 and 6 is due to the simultaneous shadowgraph. Note that the color range is saturated to visualize the doublets more clearly. The recorded images themselves are not saturated

undistorted (frame 8). This scenario is consistent with the earlier predictions (Fig. 1, negative $\theta$ ).

Besides being blurred, the particle image in frame 7 is also subject to a concurrent position error, which can be seen by comparing it to the true particle position. The true particle is in fact at the same location in the frames $1-7$, because the flow upstream of the shock is at rest. Therefore, it can be determined from the undistorted part of the particle images (frames 1-6). The black solid line in Fig. 4 connects their peaks, thus marking the true particle location. By comparison, the blurred particle image in frame 7 appears shifted to the left, in the direction of negative $s$, as expected. The position error is approximately $-6.5 \mu \mathrm{m}$ based on the peak intensity location, which corresponds to an apparent velocity error between frames 6 and 7 of
1

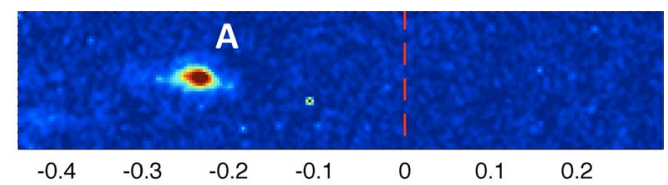

2

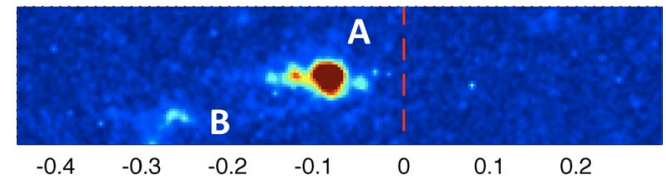

3

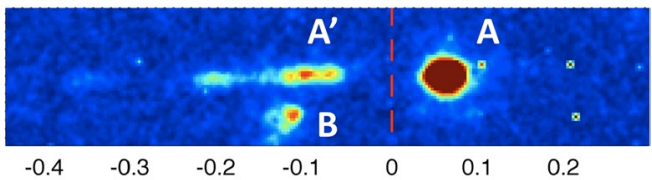

4

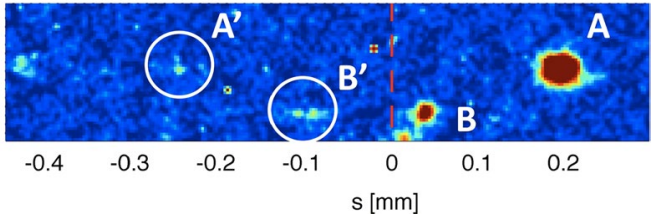

Fig. 5 A time series showing reflection-type doublets. $A^{\prime}$ and $B^{\prime}$ indicate the mirrored particle images of particles $A$ and $B$, respectively. The red dashed line indicates the shock position. Note that the color range is saturated to visualize the doublets more clearly. The recorded images themselves are not saturated

$22 \mathrm{~m} / \mathrm{s}$ toward the left (decreasing the observed particle velocity relative to the shock by about $5 \%$ ). After crossing the shock, the position error disappears and the particle image returns approximately to its original position on the black solid line. The particle motion apparently was not yet affected by the shock passage. Consequently, the resulting velocity error between frames 7 and 8 is $22 \mathrm{~m} / \mathrm{s}$ to the right. When considering the particle velocity relative to the shock, $V_{s}$, these errors result in a negative relative velocity error, $\varepsilon_{s}$, followed by a positive $\varepsilon_{s}$ consistent with Fig. 2.

Evidence of reflection-type doublets is presented in Fig. 5. As the particle labeled A crosses the shock and moves into the high-density region, a mirror image appears on the low-density side of the shock (frame 3), which can only be explained by reflection, and not by refraction. The stretching of the mirrored image A' may be caused by some shock curvature or boundary effects of the shock wave at the test section window. Furthermore, when A is just upstream of the shock, its image reveals two intensity peaks (frame 2). This suggests part of the light scattered by this particle intersects with the shock resulting in a refraction-type doublet. In the last frame, we may again observe mirrored images $\mathrm{A}^{\prime}$ and $\mathrm{B}^{\prime}$ of the particles $\mathrm{A}$ and $\mathrm{B}$. This is subject to some uncertainty as these intensity peaks are only just above the noise level. In summary, the different types of optical distortion (Fig. 1) can all be observed experimentally. 


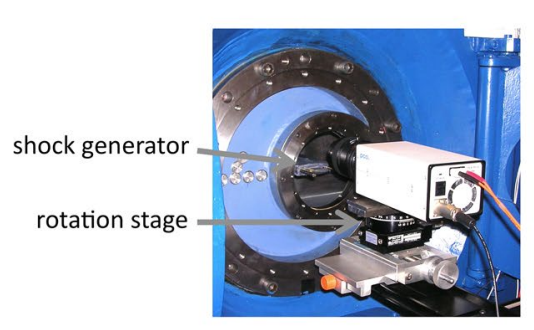

Fig. 6 Photograph of the experimental setup (left) and the measured particle velocity within the field of view (right). The camera can be rotated in the horizontal plane in order to change the viewing direc-

\section{Velocity error: experiment}

An experimental assessment of the velocity error is made based on PIV experiments performed in the TST-27 transonic-supersonic wind tunnel of Delft University of Technology in the Aerospace Engineering Department. The experimental setup was similar to the numerical simulation (Fig. 1). The stationary shock wave was generated from a 2D wedge-plate model spanning the full width of the test section (Scarano and Van Oudheusden 2003). The free-stream flow expanded from 1.94 bars stagnation pressure at ambient temperature to Mach 1.96, yielding a free-stream velocity of $500 \mathrm{~m} / \mathrm{s}$ and density of $0.56 \mathrm{~kg} / \mathrm{m}^{3}$ upstream of the shock. The wedge with sharp leading edge imposed a flow deflection of $11.3^{\circ}$ resulting in an oblique planar shock wave at an angle of $41^{\circ}$ with respect to the free stream. Over the shock, the flow velocity decreased to $440 \mathrm{~m} / \mathrm{s}$ and the density increased to $0.86 \mathrm{~kg} / \mathrm{m}^{3}$.

The flow was seeded with $50-\mathrm{nm} \mathrm{TiO}_{2}$ particles, which are known to form agglomerates of much larger size (Scarano and Van Oudheusden 2003; Ragni et al. 2011). The particles were illuminated by a double-pulse Nd:YAG laser (400 mJ per pulse) in a 1-mm-thick light sheet at the center of the test section. The distance between the sheet and the tunnel window, $W$, was $140 \mathrm{~mm}$. A 12-bit CCD camera equipped with a Nikon $60-\mathrm{mm}$ objective was used to record the images at $1376 \times 432$ pixels resolution corresponding to a field of view of $35 \times 11 \mathrm{~mm}^{2}(M=0.25)$. The camera could be translated and rotated in the horizontal plane to control the viewing direction (Fig. 6). Therefore, instead of changing the flow, i.e., the orientation of the shock within the test section as in the numerical simulation, the imaging optics were moved, which is equivalent for small viewing angles. The viewing angle with respect to the plane of the shock is obtained from the goniometric relation $\sin \theta=\sin \sigma \sin \theta_{x}$, in which $\sigma=41^{\circ}$ is the shock angle with the horizontal direction and $\theta_{x}$ is the angle of rotation of the camera in the horizontal plane (Fig. 6). In this experiment, the horizontal direction coincides with $x$, tion relative to the plane of the shock $\theta$. The camera rotation angle in the horizontal plane is given by $\theta_{x}$

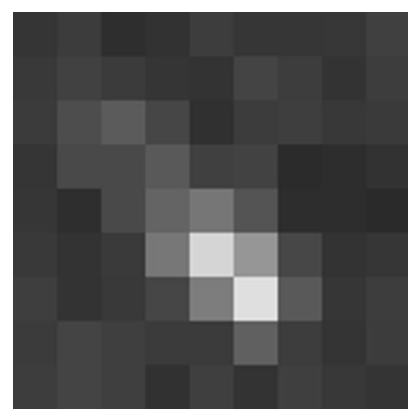

Fig. 7 Skewed directional blur observed in a real PIV recording

the direction of the free stream. The time separation was set at $0.6 \mu$ s yielding a 12-pixel particle displacement in the free stream. For each test case, the dataset consisted of 300 recordings, which were analyzed by cross-correlation using a final interrogation window size of $31 \times 31$ pixels, corresponding to $0.79 \times 0.79 \mathrm{~mm}^{2}$, at $50 \%$ overlap. An example of a blurred particle image is presented in Fig. 7. The image is stretched in the direction normal to the shock, which is at a $41^{\circ}$ angle with the horizontal in this case, and is skewed with the tail pointing in the direction of the lowdensity side, as in the simulation (Fig. 1b).

Profiles of the measured average velocity component, $V_{s}$, were extracted along a single line in the direction normal to the shock, $s$. The results are given in Fig. 8a for viewing directions, $\theta$, ranging from $-6.6^{\circ}$ to $+6.6^{\circ}$ and with $f / 16$. The velocity is normalized using the upstream value of $V_{s}$. The profiles are compared with the reference profile obtained with the viewing direction aligned with the shock, in which case the area affected by aero-optical distortion is so small that its influence on the velocity cannot be discerned due to insufficient spatial resolution. This reference profile displays the well-known exponential decay of the velocity downstream of the shock, which is associated with particle relaxation (Melling 1997; Urban and Mungal 2001; Scarano and Van Oudheusden 2003; Schrijer and Scarano 2007). The other velocity profiles are corrupted by a wiggle 

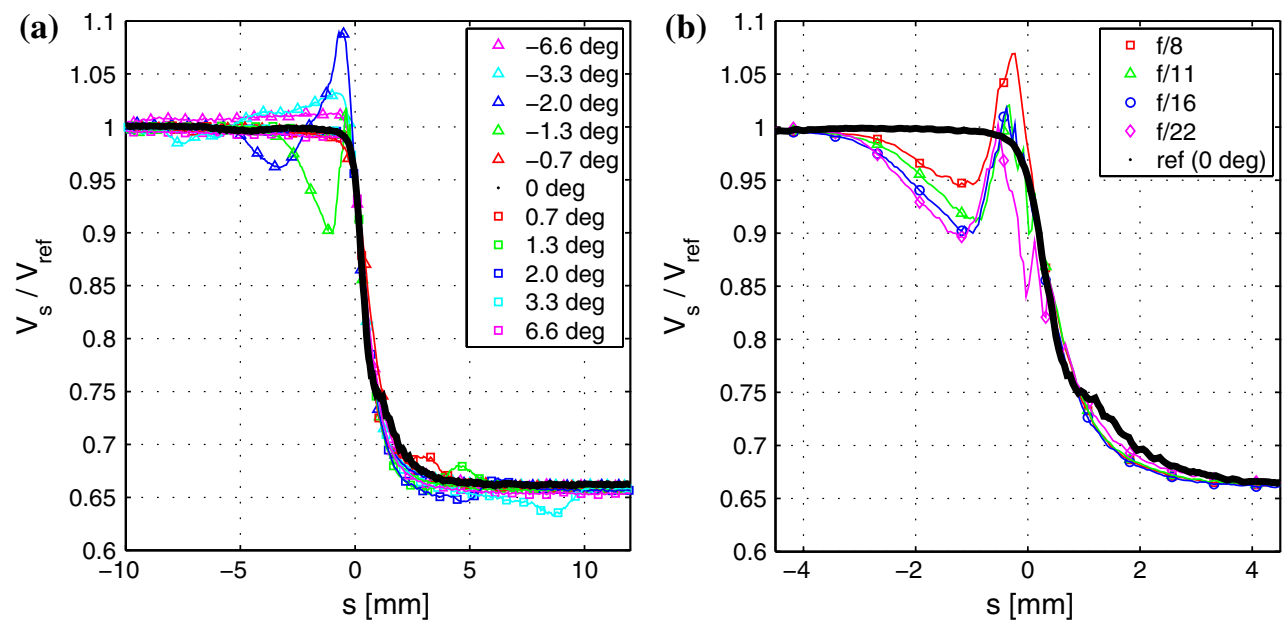

Fig. 8 Effect of the viewing direction $\theta$ (a) and the $f / \#(\mathbf{b})$ on the measured average velocity profiles across a shock wave. The viewing direction was varied at constant $f / 16(\mathbf{a})$, while $\theta=-1.3^{\circ}$ in (b). The black line indicates the reference profile obtained at $\theta=0^{\circ}$ and $f / 16$

relative to the reference profile suggesting unphysical particle accelerations, which is consistent with the predictions illustrated in Fig. 2. The affected area shifts from the pre-shock region $(s<0)$ to the post-shock region $(s>0)$ when going from negative viewing angles (looking from the high-density side) to positive angles, and the affected area increases with the magnitude of the viewing angle, as expected. The velocity error increases with decreasing magnitude of the angle until the spatial extent of the distortion features drops below the spatial resolution limit of the present PIV analysis making the error vanish in the results (angles below $0.7^{\circ}$ ). Therefore, the most critical angles for the present velocity measurement are between $-1.3^{\circ}$ and $-2.0^{\circ}$, where velocity errors up to $\pm 10 \%$ are observed relative to the reference profile. Indeed, the error reduces considerably to around 3 and $1 \%$ when using $\theta=-3.3^{\circ}$ and $-6.6^{\circ}$. The larger magnitude angles, however, suffered from perspective errors associated with varying image magnification across the field of view, which had to be corrected for. For positive viewing angles (looking from the low-density side), the error generally tends to be smaller at $4 \%$ of the local velocity. However, errors in the downstream region of the shock would disrupt an accurate measurement of the particle response to the shock, which is often the purpose of such measurements. Overall, the order of the velocity error is in line with the predictions made in Sect. 2. The remaining quantitative differences may be attributed to the $f / \#$, the particle displacement and the correlation window size all being finite in the experiments, as well as to shock-wave boundary layer interaction over the tunnel window.

The $f / \#$ is found to have a clear effect on the average velocity profiles as well (Fig. 8b). A decreasing f/\# means the light cone captured by the imaging optics is increased resulting in the particle image being affected by a larger region within the flow. This can have a smoothing effect reducing the particle position errors, hence the velocity errors. Note, however, that reducing $f / \#$ will increase the geometrical particle image blur (Eqs. 5, 6). The average velocity profiles at $s=-1.2 \mathrm{~mm}$ (the location of the undershoot in Fig. 8b) confirm the decrease in velocity error magnitude with decreasing $f / \#$. The subsequent overshoot in the average velocity, corresponding to positive $\varepsilon_{s}$, at around $s=-0.2 \mathrm{~mm}$ reveals the opposite behavior. The width of this peak in the distorted velocity profile is comparable to the cross-correlation window size $(0.8 \mathrm{~mm})$ and is not well resolved. According to the above argument, the velocity error increases with increasing $f / \#$, which will cause the particle displacements to vary by more than $10 \%$ within a correlation window when $f / \#>11$, as may be inferred from Fig. 8. Clearly, the correlation analysis is not able to cope, which is evident from the sharp oscillations visible in the velocity profile, especially for $f / 22$. Moreover, the rms of the normalized streamwise component of velocity peaks at the same location, $s=-0.2 \mathrm{~mm}$ (Fig. 9b). Since the flow is steady, the rms directly represents the measurement noise level, which increases from 0.1 (for $f / 8$ and reference case) to 0.45 (f/22). The increase in noise cannot be associated with image blur, since the latter is actually decreasing with $f / \#$, but must be attributed to the very strong, unresolved velocity gradients introduced by the optical distortion position error near $s=-0.2 \mathrm{~mm}$. These gradients indeed increase with $f / \#$. The rms is thus dominated by spurious peak detection in consequence of a low signal-to-noise ratio in the correlation maps.

It is noted that, even in the absence of important optical distortion errors, the rms velocity increases at the shock 

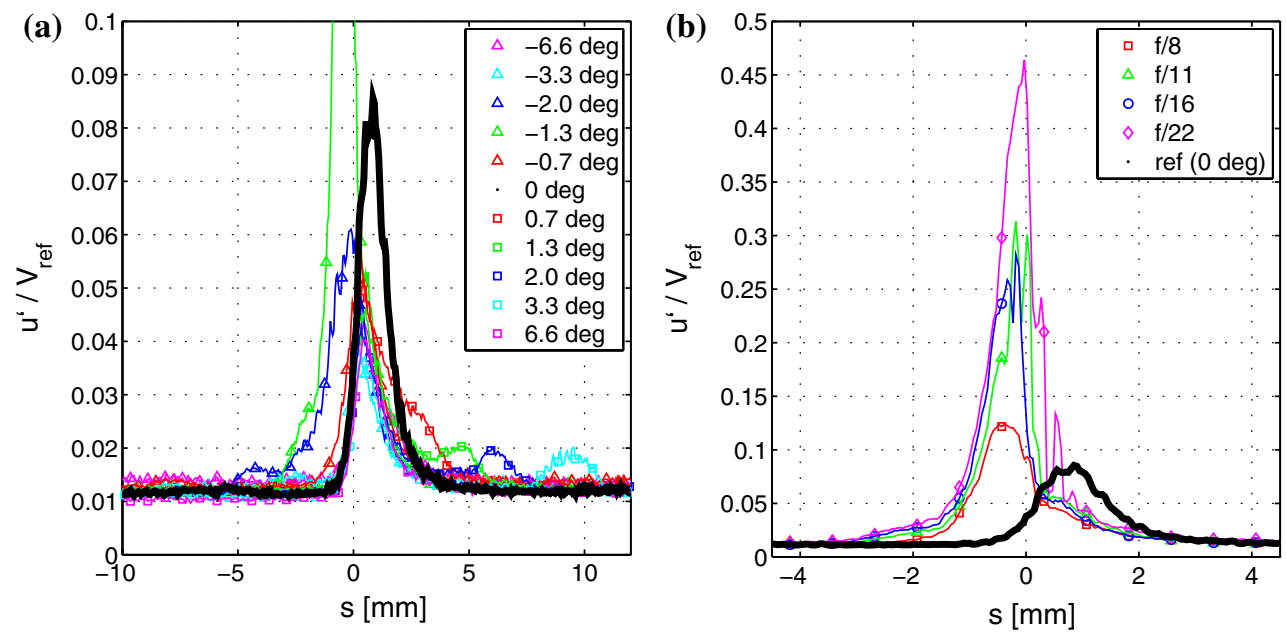

Fig. 9 The rms of the streamwise velocity component $u^{\prime}$ for varying $\theta$ at $f / 16$ (a) and for varying $f / \#$ at $\theta=-1.3^{\circ}$ (b). The black line indicates the reference profile obtained at $\theta=0^{\circ}$ and $f / 16$
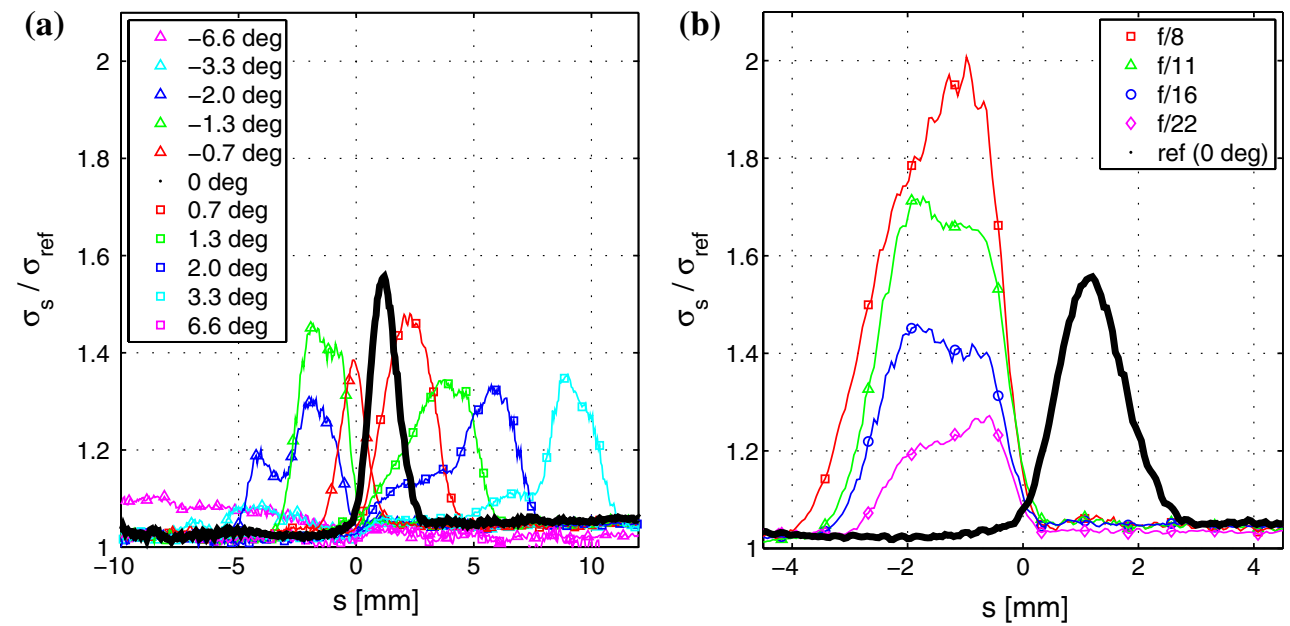

Fig. 10 Width of the autocorrelation peak in the direction $s$ normal to the shock for varying $\theta$ at $f / 16$ (a) and for varying $f / \#$ at $\theta=-1.3^{\circ}(\mathbf{b})$. The black line indicates the reference profile obtained at $\theta=0^{\circ}$ and $f / 16$

position, which is due to the sharp change in the particle velocity locally (Mitchell et al. 2011). Presently, this background increase is estimated at only 0.04 at $s=0$, which is inferred from the cases without significant optical distortion (Fig. 9a, cases $\theta= \pm 6.6^{\circ}$ ). The background increase is clearly less than the peaks in rms velocity in Fig. 9b, which therefore must be ascribed to the optical distortions as discussed above.

Figure $8 \mathrm{~b}$ also reveals that even the reference profile at $\theta=0$ contains small velocity errors. Due to the negative angles $\theta$, the other profiles are affected by distortion only upstream of the shock. Their particle relaxation tail can thus be considered accurate. The reference profile, however, oscillates about their tail for $s$ between 0.5 and $2.5 \mathrm{~mm}$, as will be shown more clearly in Sect. 5 .
As mentioned before, blur and particle position error are concurrent. While the latter may not always be straightforward to detect, particle image blur can be established directly by an autocorrelation analysis of the images using the same correlation windows as before in the PIV crosscorrelation analysis. If blur occurs within the correlation window, the autocorrelation peak will be elongated similar to the elongated particle images. Figure 10 presents the width of the average autocorrelation peak across the shock. The peak width is quantified by the standard deviation $\sigma_{s}$ of the Gaussian peak fit in the direction of blur, which coincides with $s$. An increase in peak width is indeed visible in the region where optical distortion errors are observed (compare Figs. 8, 10), including the reference case. Blur can therefore be used as a detector for possible velocity 
Fig. 11 Particle relaxation length, $\chi_{p}$, determined from a fit (dashed line, red) to the measured particle velocity response profile, $u^{*}$ (solid line, black). Results are presented for different viewing directions $\theta$ at constant $f / 16$
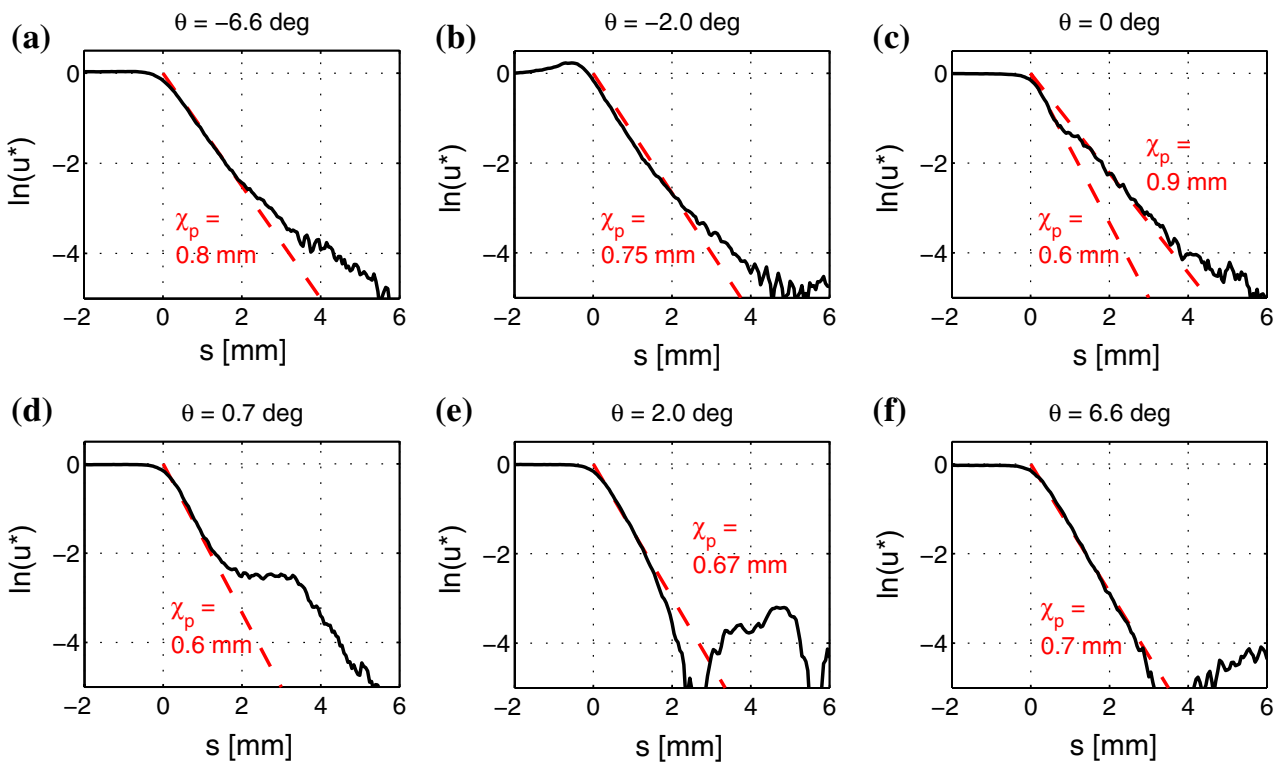

error near shocks. However, attempts at quantifying the velocity error based on image blur detection have remained unsuccessful so far.

\section{Particle response measurements}

Shock tests are typically used to measure the particle response to a step change in flow velocity. Therefore, in this section, we illustrate how the aero-optical velocity error affects such measurements. The assessment is based on the particle relaxation length determined from the PIV data presented in the previous section.

The temporal response of a small particle to a shock is given by an exponential decay in the particle's velocity (Melling 1997), where the initial and the final particle velocities are equal to the upstream and the downstream flow velocity, respectively. This result was derived from the equation of particle motion in the assumption that the density of the particle is large compared to the density of the fluid. Rewritten in terms of a natural logarithm, the particle velocity decay is given by:

$\ln \left(u^{*}\right)=\ln \left(\frac{V_{s}-u_{s 2}}{u_{s 1}-u_{s 2}}\right)=-\frac{t}{\tau_{p}}$

where $u_{s}$ is the flow velocity component normal to the shock with the indices 1 and 2 referring to the conditions upstream and downstream of the shock. $V_{s}$ is the particle velocity in the direction normal to the shock, as before. Its decay is characterized by a single time constant $\tau_{p}$, which is known as the particle relaxation time. The temporal response is converted to the spatial response using a constant velocity scale, i.e., the upstream velocity $u_{s 1}$. The constant velocity approximation introduces a small error
(Ragni et al. 2011), but it is the same error in all cases and therefore not relevant to the present assessment of the aerooptical distortion effects. The spatial decay profile of $u^{*}$ is then approximated as:

$\ln \left(u^{*}\right) \cong-\frac{s}{\chi_{p}}$

where $\chi_{p}$ is the relaxation length. The relaxation length can now be easily determined from the slope in a plot of $\ln \left(u^{*}\right)$ versus the shock normal distance $s$ (Schrijer and Scarano 2007; Ragni et al. 2011).

Profiles of $\ln \left(u^{*}\right)$ obtained from the PIV measurements (Sect. 4) are presented in Fig. 11. Directly downstream of the shock $(s>0)$, the particle velocity is expected to follow an exponential decay according to Eq. 8, which appears in these graphs as a straight line with a negative slope $-1 / \chi_{p}$. For a moderate negative viewing angle $\left(\theta=-6.6^{\circ}\right)$, the velocity measurement downstream of the shock is not affected by optical distortion, and the error upstream of the shock remains small as discussed in Sect. 4. Therefore, this case is used to establish a reference particle relaxation length, which is not affected by aero-optical distortion. The corresponding profile (Fig. 11a) indeed shows the initial linear decay of $\ln \left(u^{*}\right)$ downstream of the shock between $s=0$ and $2 \mathrm{~mm}$. The slope in this range is determined from a linear fit (dashed line), which yields a relaxation length $\chi_{p}$ of $0.80 \mathrm{~mm}$ corresponding with a relaxation time $\tau_{p}$ of $2.4 \mu \mathrm{s}$. The particle relaxation time is in agreement with the reported value by Scarano and Van Oudheusden (2003) for the same seeding material. Note that $\chi_{p}$ is approximately equal to the correlation window size and that $\tau_{p}$ is about four times the pulse separation time, which complies with the conditions for a resolved measurement of the particle response according to Ragni et al. (2011). 
When changing the viewing direction, the measurement of the particle relaxation length is affected in different ways. For $\theta=-2.0^{\circ}$ (Fig. 11b), the optical distortion of the measured velocity profile is still upstream of the shock, as discussed in Sect. 4, but the velocity overshoot makes it more difficult to precisely determine the shock location and the upstream particle velocity from the plot. These complicating factors contribute to the initial velocity decay $(0<s<2 \mathrm{~mm})$ not revealing a very clear exponential behavior as compared to Fig. 11a. A fit in this region yields $\chi_{p}=0.75 \mathrm{~mm}$, which is close to the reference value $\left(\chi_{p}=0.80 \mathrm{~mm}\right)$. Then for $\theta=0$ (Fig. 11c), two slopes may be identified. The initial decay follows $\chi_{p}=0.60 \mathrm{~mm}$ before settling to $\chi_{p}=0.90 \mathrm{~mm}$. In consequence, the uncertainty in determining $\chi_{p}$ is large. Finally for positive angles $\theta$, the velocity errors are in the downstream region of the shock, which directly affects the measured particle relaxation as seen in Figs. 11d-f. The resulting relaxation lengths $\chi_{p}$ are $0.60,0.67$ and $0.70 \mathrm{~mm}$ for $\theta=0.7^{\circ}, 2.0^{\circ}$ and $6.6^{\circ}$, respectively. Relative to the reference value, this corresponds with 25,16 and $13 \%$ errors in $\chi_{p}$, which follows the general trend of decreasing velocity error with increasing $\theta$ (Sect. 2). The basic fact that the $\theta=6.6^{\circ}$ case (Fig. 11f) reveals a clear exponential decay does not mean it is free of error. Moderate negative viewing angles $\left(\theta \approx-5^{\circ}\right)$ are thus recommended when measuring particle relaxation.

\section{Conclusion}

Light ray tracing, PTV and PIV experiments were used to assess the effects of aero-optical distortions associated with the imaging of particles through planar shock waves. The shock in this case presents an optical interface over which the refractive index shows a jump. Results revealed skewed particle image blur in the direction normal to the shock and two types of particle image doublets, which were caused either by refraction or by reflection of light at the location of the shock. The latter type of doublet appeared to move in the opposite (i.e., mirrored) direction to the actual flow. Moreover, particle image blur was found to be concurrent with an error in the particle position within the images, which in turn gave rise to significant errors when evaluating the particle's velocity. Hence, blur marks position and velocity error. These errors were shown to depend strongly on the viewing angle $\theta$, but experiments also revealed a certain dependence on the $f / \#$. The results demonstrated that increasing this angle $\theta$ is a particular effective way to decrease the relative velocity error. If the accurate measurement of the particle response to the shock wave is desired, it is recommended to look at the shock from the high-density side at a moderate angle $\left(\theta \approx-5^{\circ}\right)$ as the optical distortions affect only the upstream flow region.

The order of the velocity error predicted from ray tracing agreed with the errors observed in the wind tunnel tests, but quantitative details would depend on the exact shock geometry, which was likely affected by shock-boundary layer interactions occurring at the wind tunnel window. A major source of PIV measurement noise was found to be the sharp velocity gradients introduced by the optical distortion, whereas the correlation peak broadening by the blurred particle images was found to be only a minor source of PIV measurement uncertainty. Therefore, it is favorable to use a relatively small $f$ /\# to measure shock waves because of the smoothing effect on the position and the velocity error, even though it increases the particle image blur.

Acknowledgments The helpful comments by Prof. F. Scarano and Drs. B.W. van Oudheusden and F.F.J. Schrijer are gratefully acknowledged. Thanks to Dr. K. Prestridge at Los Alamos National Laboratory for providing experimental validation data.

Open Access This article is distributed under the terms of the Creative Commons Attribution 4.0 International License (http://creativecommons.org/licenses/by/4.0/), which permits unrestricted use, distribution, and reproduction in any medium, provided you give appropriate credit to the original author(s) and the source, provide a link to the Creative Commons license, and indicate if changes were made.

\section{References}

Adamson TC, Messiter AF (1980) Analysis of two-dimensional interactions between shock waves and boundary layers. Annu Rev Fluid Mech 12:103-138

Buchmann NA, Cierpka C, Kähler CJ, Soria J (2014) Ultra-highspeed 3D astigmatic particle tracking velocimetry: application to particle-laden supersonic impinging jets. Exp Fluids 55:1842

Elsinga GE, van Oudheusden BW, Scarano F (2005) Evaluation of aero-optical distortion effects in PIV. Exp Fluids 39:246-256

Hou YX, Clemens NT, Dolling DS (2002) Development of a multicamera piv imaging system for studies of shock/boundary layer interactions. In: 22nd AIAA aerodynamic measurement technology and ground testing conference. St. Louis, MO, USA, AIAA-2002-3232

Martinez AA, Orlicz GC, Prestridge KP (2015) A new experiment to measure shocked particle drag using multi-pulse particle image velocimetry and particle tracking. Exp Fluids 56:1854

Melling A (1997) Tracer particles and seeding for particle image velocimetry. Meas Sci Technol 8:1406-1416

Merzkirch W (1974) Flow visualization. Academic Press, London

Mitchell D, Honnery D, Soria J (2011) Particle relaxation and its influence on the particle image velocimetry cross-correlation function. Exp Fluids 51:933-947

Murphy MJ, Adrian RJ (2010) PIV space-time resolution of flow behind blast waves. Exp Fluids 49:193-202

Petersen EL, Hanson RK (2003) Improved turbulent boundary-layer model for shock tubes. AIAA J 41:1314-1322

Raffel M, Kost F (1998) Investigation of aerodynamic effects of coolant ejection at the trailing edge of a turbine blade model by PIV and pressure measurements. Exp Fluids 24:447-461 
Raffel M, Willert C, Kompenhans J (1998) Particle image velocimetry: a practical guide. Springer, Berlin, p 137

Ragni D, Schrijer F, van Oudheusden BW, Scarano F (2011) Particle tracer response across shocks measured by PIV. Exp Fluids 50:53-64

Scarano F, Van Oudheusden BW (2003) Planar velocity measurements of a two-dimensional compressible wake. Exp Fluids 34:430-441

Schrijer FFJ, Scarano F (2007) Particle slip compensation in steady compressible flows. In: 7th International symposium on particle image velocimetry, Rome, Italy
Schrijer FFJ, Scarano F, Van Oudheusden BW, Bannink WJ (2005) Application of PIV in a hypersonic double-ramp flow. In: 13th AIAA/CIRA International Space Planes and Hypersonic Systems and Technologies Conference. Capua, Italy, AIAA-2005-3331

Settles GS (2001) Schlieren and shadowgraph techniques: visualizing phenomena in transparent media. Springer, Berlin

Urban WD, Mungal MG (2001) Planar velocity measurements in compressible mixing layers. J Fluid Mech 431:189 